\title{
A novel phase congruency based algorithm for online data reduction in ambulatory EEG systems
}

\author{
Lojini Logesparan, Student Member, IEEE, and Esther Rodriguez-Villegas, Senior Member, IEEE
}

\begin{abstract}
Real signals are often corrupted by noise with a power spectrum variable over time. In applications involving these signals, it is expected that dynamically estimating and correcting for this noise would increase the amount of useful information extracted from the signal. One such application is scalp EEG monitoring in epilepsy, where electrical activity generated by cranio-facial muscles obscure the measured brainwaves. This paper presents a data selection algorithm based on phase congruency to identify interictal spikes from background EEG; together with a novel statistical method that allows a more comprehensive trade-off based quantitative comparison of two algorithms which have been tested at a fixed threshold in the same database. Here, traditional phase congruency has been modified to incorporate a dynamic estimate of muscle activity present in the input scalp EEG signal. The proposed algorithm achieves $50 \%$ data reduction whilst detecting more than $80 \%$ of interictal spikes. This represents a significant improvement over the state-of-the-art denoising method for phase congruency.
\end{abstract}

Index Terms-EEG, epilepsy, online data reduction, phase congruency, spike detection.

\section{INTRODUCTION}

$\mathbf{E}$ PILEPSY is a common neurological disorder that affects 50 million people worldwide [1]. Electroencephalography (EEG) is a key tool for diagnosing the condition. The benefits of long term ambulatory EEG (AEEG) monitoring for both diagnosis and treatment have been extensively reported [1]-[3]. Unfortunately there is often a lack of in-patient resources, which, in addition to limitations of current EEG systems for out-patient monitoring, makes long term monitoring only feasible for patients with refractory epilepsy [1]. During ambulatory EEG monitoring, a patient is tethered to a batterypowered EEG system for days or even weeks. Although it is portable, the device is still too bulky and heavy for the patient to carry out routine activities [4]. A potential solution is to miniaturize the AEEG system by wireless transmission of the monitored signals to a base station [4]. However wireless transmission is power hungry and can hence also impact the overall size of the system [5]. Two possible methods to reduce the power consumption of such a system are: to customize each of the electronic blocks within the system [6]; or reduce the amount of data transmitted.

This paper proposes a novel data selection algorithm based on phase congruency to identify candidate interictal activity

Manuscript received October 21, 2010; revised January 21, 2011 and April 05, 2011. Copyright (c) 2010 IEEE. Personal use of this material is permitted. However, permission to use this material for any other purposes must be obtained from the IEEE by sending an email to pubs-permissions@ieee.org. The research leading to these results has received funding from the European Research Council under the European Community's 7th Framework Programme (FP7/2007-2013) / ERC grant agreement no. 239749.

L. Logesparan and E. Rodriguez-Villegas are with the Department of Electrical and Electronic Engineering, Imperial College London, SW7 2AZ. e-mail:\{lojini.logesparan04, e.rodriguez\}@imperial.ac.uk and reject background (normal) activity of the brain. The algorithm is compared with the state-of-the-art denoised phase congruency technique, and a new method to statistically validate the difference in performance of the two algorithms is also proposed. Phase congruency, an image processing technique to detect edges and lines [7], is used to identify interictal spikes (interictal activity consists of spikes, sharp-waves and spikeand-waves henceforth collectively referred to as spikes). Phase congruency has many applications in image processing [8], [9] and it has also been tested for epileptic seizure detection on EEG signals [10]. The technique extracts phase information from different frequency components of the input signal, by calculating the local energy and amplitude of the frequency components at any time instance [11]. This method has been later modified to include an estimate of noise. The most-cited denoising method [7] (henceforth referred as denoised phase congruency (DPC)) developed for image denoising, assumes a constant power spectrum which is not true for many real signals such as scalp EEG. When an EEG signal is recorded on the scalp of the patient, the main interference is the electrical activity generated by the contraction and expansion of craniofacial muscles. The latter obscures the recorded EEG making it unworthy to the neurologist. This work proposes an alternative technique to [7], to denoise phase congruency calculations by dynamically estimating and correcting for the cranio-facial muscle activity present in scalp EEG.

This paper presents traditional phase congruency, the mostcited denoising method and the proposed modified phase congruency (MPC) in Section II. Modified phase congruency has been incorporated into a data selection algorithm to identify spikes. A preliminary version of this algorithm has been presented in [12]. In this paper, a larger patient database has been used to test the three phase congruency based algorithms. Different metrics and results have been derived in Section III. Finally, Section IV describes a new method to compare and statistically validate the performance of two algorithms tested on the same database. This technique has been applied to denoised and modified phase congruency algorithms, to decide which algorithm would perform better when implemented in hardware.

\section{Methods}

In an EEG system, discontinuous recording of the input EEG signal could achieve high data reduction in records where spikes are rare [5]. Hence, designing a data selection algorithm that aims to detect candidate spikes -or in different words, eliminates everything that is with a high degree of certainty not useful diagnosis data- could, in a wireless system, allow for the transmitter to be turned off when there are no detections.There are potential advantages associated to this 


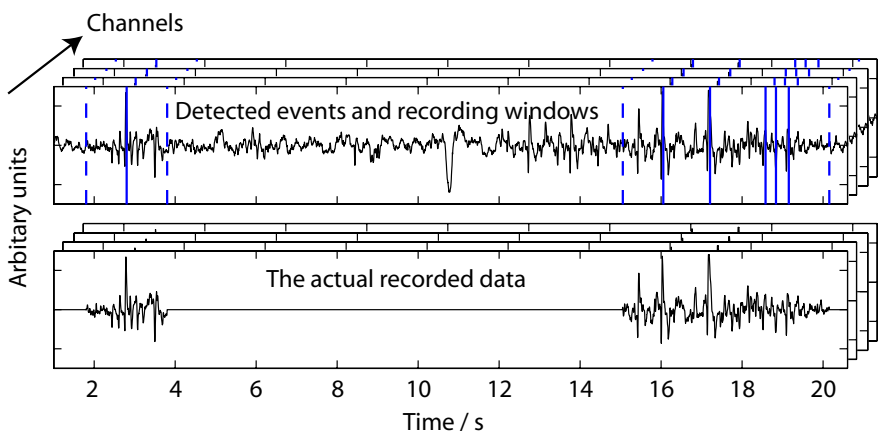

Fig. 1. Multi-channel EEG showing detected candidate events and the recorded data [13]

approach which can be summarized as: 1- Power saving due to the shorter transmission times, which could translate into longer operating lifetimes from smaller batteries/systems. 2Reduction in the amount of diagnostically irrelevant data as well as diagnostic time, whilst still leaving the role of diagnosis to the neurologists. 3- Implementation simplification, as false detections are not as critical as in algorithms that aim for automatic diagnosis. However, there is an added technical challenge versus algorithms for automatic diagnosis: whereas an automatic detection algorithm can be run in the backend and hence complexity is not a problem, data selection has to happen in the front-end before transmission. Because of this, complexity needs to be kept to a minimum so that the overhead in terms of power consumption of the hardware implementation is significantly lower than the potential power saved by the reduction in data transmission [13].

A data selection algorithm has been designed to identify and select for transmission candidate spikes, and its principle is shown in Fig. 1 [13]. The algorithm analyzes each EEG channel separately. When an interictal spike is detected in a single channel, a short duration of data before and after the detected spike, called the recording window, is selected for transmission. The additional background data on either side of the candidate spike is useful for neurologists to diagnose an interictal spike from the transmitted candidate events. It should be noted that the data selection algorithm purely selects candidate spikes with the intention of reducing the amount of data transmitted, hence the diagnosis of an interictal spike still remains as the role of the neurologist. On detection, this recording window of data is transmitted across all channels. When there are no detections, no data will be transmitted.

\section{A. Phase congruency}

The proposed data selection algorithm is based on phase congruency. Phase congruency is an image processing technique developed to detect edges and lines [7]. Fourier components of the image at edges or lines will be at maximum phase [7]. The same can be expected at sharp transients, such as spikes. An interictal spike can be visually identified amongst background EEG, as a transient with a duration ranging from less than $70 \mathrm{~ms}$ up to $200 \mathrm{~ms}$ [14]. Hence it can be predicted that the phase congruence at an interictal spike would be higher than background EEG. This difference has been utilized in designing the data selection algorithm to distinguish between candidate spikes and background.
Phase information can be extracted from an input signal using multiple wavelet filters over the wanted frequency range [7]. The filters should have linear phase to preserve the phase information from the signal. Hence, nonorthogonal wavelets are required in a symmetric/antisymmetric quadrature pair [7]. Log Gabor wavelet filters have been chosen as they have zero d.c component, which is crucial to ensure that low frequencies are not over-represented in large bandwidth even-symmetric filters [7]. In this study, traditional phase congruency is compared with the most-cited denoised phase congruency (described in [7]) hence the wavelet filters were selected a priori to match the filters in [7].

To calculate phase congruency, five log Gabor filters have been designed to have a bandwidth close to one octave, and span $1.5 \mathrm{~Hz}$ to $15.5 \mathrm{~Hz}$. The bandwidth has been selected to maximize the wanted EEG frequency range whilst ensuring that the number of filters is kept to a minimum, in the interest of saving power.

Consider an input EEG signal $I(x)$ at a time $x$ that is filtered by even-symmetric and odd-symmetric Log Gabor filters $M_{n}^{e}$ and $M_{n}^{o}$ respectively at scale $n$ (corresponding to the filter's frequency range) [7]. The output of the even and odd-symmetric filters are:

$$
\left[e_{n}(x), o_{n}(x)\right]=\left[I(x) * M_{n}^{e}, I(x) * M_{n}^{o}\right]
$$

Phase congruency can be calculated as [11]

$$
P C(x)=\frac{E(x)}{\sum_{n} A_{n}(x)+\epsilon}
$$

where $\epsilon$ is a small value dependent on the precision of computation [7]. $A_{n}(x)$ is the sum of amplitudes across all $n$ scales

$$
\sum_{n} A_{n}(x) \simeq \sum_{n} \sqrt{e_{n}(x)^{2}+o_{n}(x)^{2}},
$$

and $E(x)$ is given by

$$
E(x)=\sqrt{F(x)^{2}+H(x)^{2}},
$$

where $F(x)$ and $H(x)$ are the sum of the output of the even and odd filters respectively

$$
\begin{aligned}
& F(x) \simeq \sum_{n} e_{n}(x) \\
& H(x) \simeq \sum_{n} o_{n}(x)
\end{aligned}
$$

\section{B. Interference in phase congruency calculations}

For the sake of clarity within the context of this paper, we will differentiate between two main sources of interference for phase congruency calculations: background activity and electrical artifacts such as the ones caused by the activity of cranio-facial muscles. In the case of background activity, it is generally understood that an ideal data selection algorithm should identify and select for transmission all interictal activity whilst rejecting all background data. However, whilst this may be the case for algorithms that are going to be integrated as part of a system for automatic diagnosis, in the situation in which diagnosis is still carried out by the neurologist, a small amount of background data is known to be not only useful 


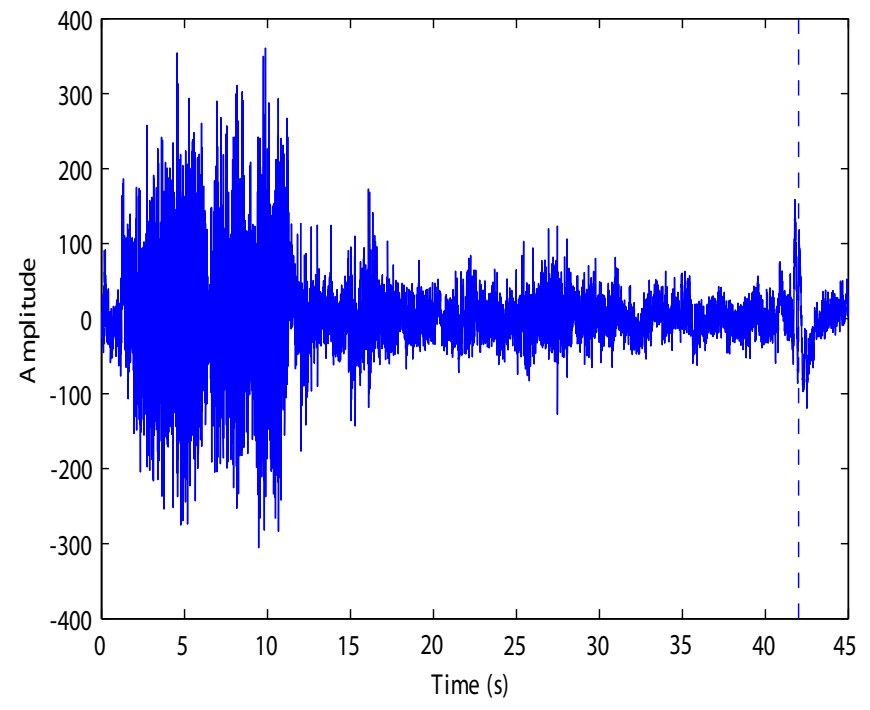

(a)

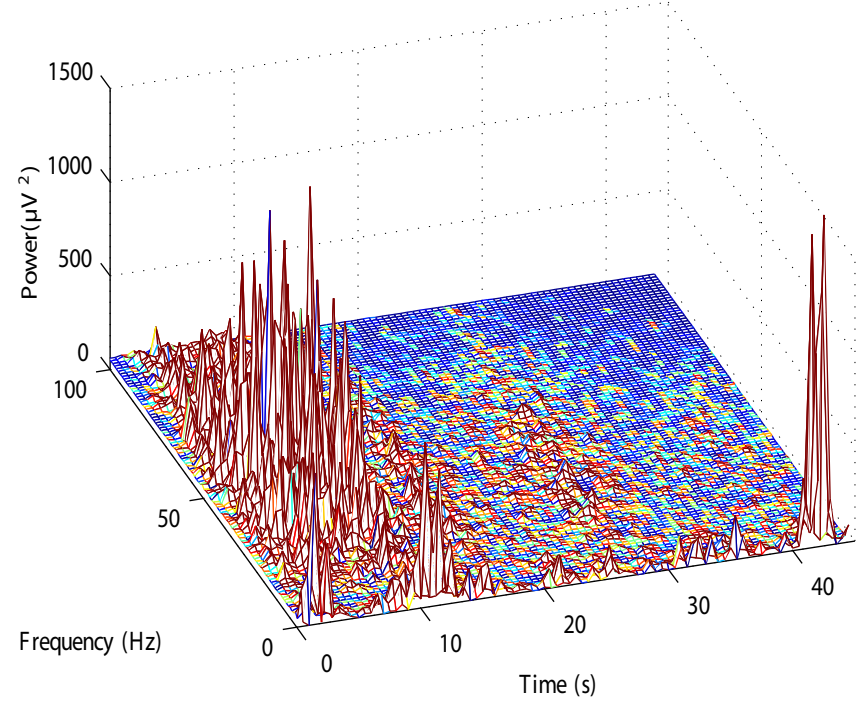

(c)

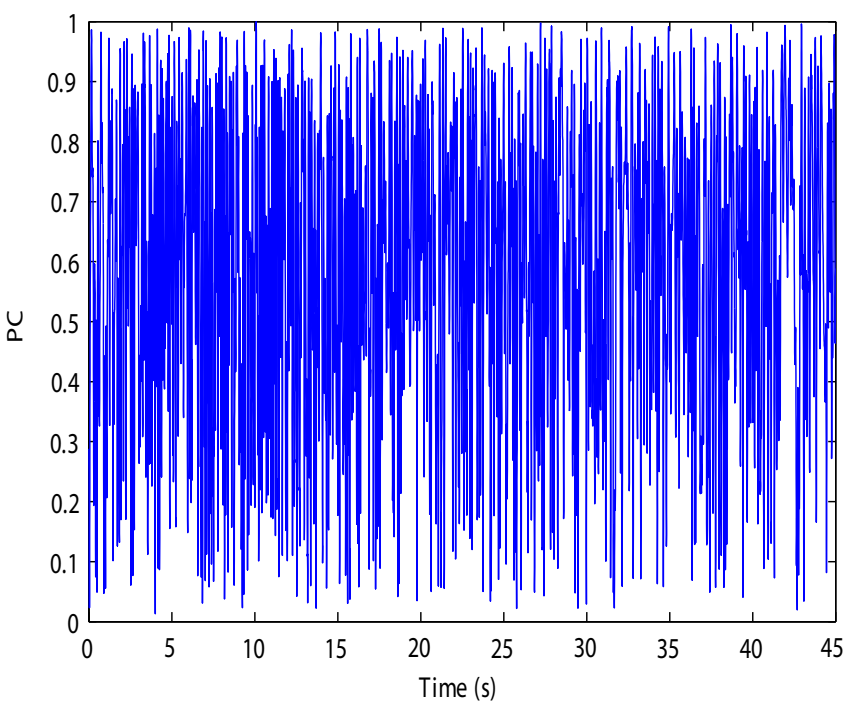

(b)

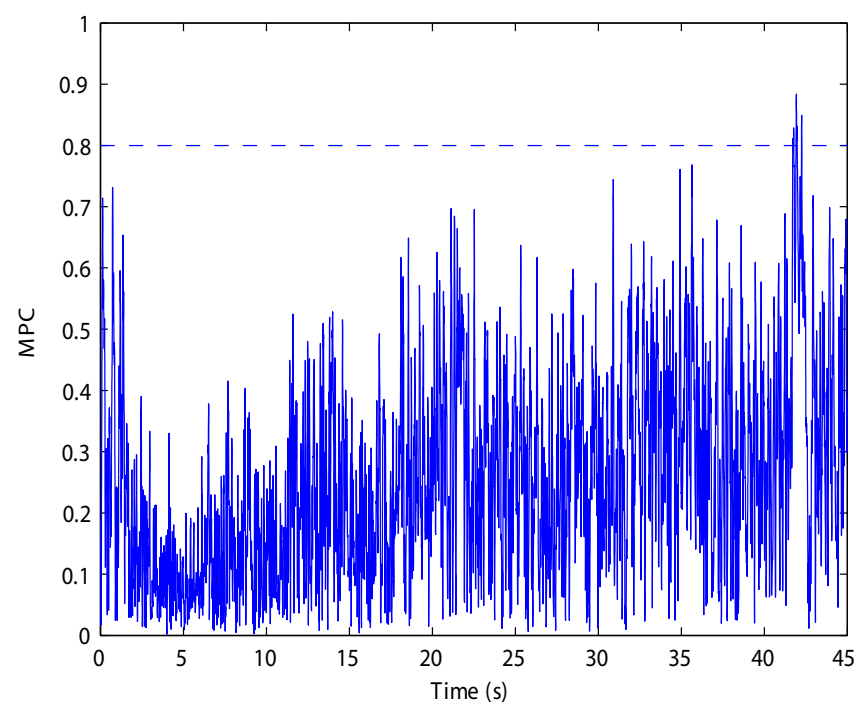

(d)

Fig. 2. Motivation for dynamic estimation and correction of higher frequency activity, in the calculation of phase congruency. High frequency muscle activity compensation in modified phase congruency allows the use of a threshold to differentiate the spike from artifacts and background activity. The threshold is represented by a dashed line in (d). (a)Scalp EEG signal with expert marked spike at 42s. (b)Traditional phase congruency. (c)Spectrogram of signal in (a). (d)Modified phase congruency

but in some cases necessary. Hence, although the amount of background data selected for transmission should be reduced in the interest of power, it is not crucial to reject all of it. On the other hand, artifacts such as those caused by muscle activity, obscure the recorded scalp EEG making it unworthy to the neurologist and hence this data should be removed prior to transmission.

A $45 \mathrm{~s}$ scalp EEG segment containing a single expert marked interictal spike at $42 \mathrm{~s}$ is shown in Fig. 2(a). The same figure also shows significant muscle activity within the first $10 \mathrm{~s}$. Traditional phase congruency as described above, has been calculated for the same EEG segment and is shown in Fig. 2(b). It can be observed how the presence of the interictal spike cannot be easily be distinguished in the calculated feature and hence denoising would be necessary for detection. In the case of artifacts caused by the contraction and expansion of cranio-facial muscles, it is known from literature that their power spectrum varies with time; is present across all frequencies; in the case of frontal and temporal muscle activities has maximum amplitudes between $20 \mathrm{~Hz}$ to $30 \mathrm{~Hz}$ and $40 \mathrm{~Hz}$ to $89 \mathrm{~Hz}$ [15]; and in general for all muscles has more power at frequencies above $15 \mathrm{~Hz}$ [16], [17]. This can be seen in the spectrogram of the raw EEG signal in Fig. 2(c) as high power across all frequencies in the first $10 \mathrm{~s}$ of the signal. Two methods could be used to remove this muscle activity: the raw EEG signal could be thresholded for high amplitude artifacts; or an estimate of the muscle activity could be incorporated into the phase congruency calculation. The problem with applying a fixed threshold to either the raw or the traditional phase congruency signals, as shown in Fig. 2(a) and Fig. 2(b) would either be the very poor performance of the method in eliminating the 
artifact related sections, or the undesired rejection of interictal spikes. On the other hand if the second method is used, it is crucial to guarantee that incorporating an estimate of muscle activity into the phase congruency calculation is not detrimental for the detection of candidate events. From the spectrogram in Fig. 2(c) it can be seen that there is more power at high frequencies until $10 \mathrm{~s}$, and similar lower power at high and low frequencies thereon until about $40 \mathrm{~s}$. In the location of the spike the power increases, but not as uniformly within the frequency spectrum, being mostly concentrated at the low frequencies. Based on this, it was hypothesized that estimating high frequency activity may not only improve phase congruency by removing sections with muscle activity, but also improve the detection of candidate spikes by reducing the phase congruence of background activity. The estimation of high frequency activity is described in Section II-D and it is compared to the most cited (state-of-the-art) method for denoising phase congruency [7], described in the next section.

\section{State-of-the-art denoising method}

The most-cited method to denoise phase congruency is described in [7] and has been tested for detecting edges in images. As it was developed for denoising images, the assumptions that have been made on image noise may not be ideal for denoising EEG signals. To our knowledge, no work has been done on denoising phase congruency for EEG signals hence we are constraint to comparing the proposed algorithm with this image denoising technique.

The method to estimate the noise level in [7] is based on a number of assumptions that can still be considered valid in the case of EEG signals:

1) Noise is additive - electrical activity generated by the cranio-facial muscles at different positions on the patient's head would be added to the brain's electrical activity when recorded using scalp electrodes.

2) Noise has a constant power spectrum - within a short time window, the power spectrum due to muscle interference may be assumed constant.

3) Features occur at isolated positions - this assumption is valid if the number of interictal spikes and their respective duration is small in comparison to the duration of the recording window.

The traditional phase congruency calculation in (2) is now modified to remove a fixed noise threshold $T$, giving denoised phase congruency [7]

$$
D P C(x)=\frac{\lfloor E(x)-T\rfloor}{\sum_{n} A_{n}(x)+\epsilon}
$$

From (4), (5) and (6) it can be derived that if the noise is Gaussian with random phase, then the wavelet filters' response is the sum of two independent normally distributed vectors which forms a Rayleigh distribution with mean $\mu_{R}$ [7] given by

$$
\mu_{R}=\sigma_{G} \sqrt{\frac{\pi}{2}}
$$

To estimate the noise response of the sum of the filters $\sigma_{G}$, it is assumed that the smallest scale filter mainly detects noise.
Thus the median of the amplitude $A_{n_{\text {min }}}$ of the smallest scale filter $n_{\min }$ [18] is

$$
\tau=\frac{\operatorname{median}\left(A_{n_{\text {min }}}\right)}{\sqrt{\ln (4)}}
$$

And the overall noise response is the sum of the individual noise contributions of each filter. This reduces to a geometric sum of the ratio of center frequencies of successive filters (mult) across $N$ number of wavelet scales [18]

$$
\sigma_{G}=\frac{\tau\left(1-\frac{1}{\text { mult }}^{N}\right)}{1-\frac{1}{\text { mult }}}
$$

Using (9) and (10), it is possible to compute the mean in (8) and also calculate the standard deviation of the Rayleigh distribution,

$$
\sigma_{R}^{2}=\left(\frac{4-\pi}{2}\right) \sigma_{G}^{2}
$$

The estimated noise threshold $\mathrm{T}$, is calculated within nonoverlapping windows of the same duration as the recording window and is given by

$$
T=\mu_{R}+2 \sigma_{R}
$$

The above description of denoised phase congruency (DPC) only aims to give the reader an idea of the technique for the purpose of comparison to the proposed method. For more details on DPC see [7] [18].

\section{Proposed modification to phase congruency}

To estimate muscle activity present in the input scalp EEG signal, log Gabor wavelet filters identical to those described in Section II-A but spanning from about $15.5 \mathrm{~Hz}$ to $64 \mathrm{~Hz}$ have been selected. The corresponding scales $m$ are calculated for 3 frequency bands using 6 additional symmetric/antisymmetric $\log$ Gabor wavelet filters. The frequency range was chosen to maximize the frequencies where muscle artifacts are present (15 Hz to $89 \mathrm{~Hz}$ as described in Section II-B), whilst minimizing the number of filters required in order to reduce the system complexity and hence the potential power consumption. The overall filter bank thus designed represents frequencies between $1.5 \mathrm{~Hz}$ to $64 \mathrm{~Hz}$ equally.

The estimate of muscle activity can now be incorporated in the phase congruency calculation in such a way that phase congruency is reduced at time instances where muscle activity is high. Hence, the amplitude of muscle activity at scales $m, A_{m}(x)$, is calculated as in (3) and incorporated in the traditional phase congruency calculation to give the modified phase congruency

$$
M P C(x)=\frac{E(x)}{\sum_{n} A_{n}(x)+\epsilon+\sum_{m} A_{m}(x)}
$$

Modified phase congruency is plotted in Fig. 2(d) for the same 45 s EEG segment as Fig. 2(b), with an expert marked spike at 42 s. In Fig. 2(d) it is clear that the spike has a higher phase congruence than the surrounding background data, and can be distinguished by applying a fixed threshold at 0.8 . In addition, it can be seen that muscle activity between $0 \mathrm{~s}$ and $10 \mathrm{~s}$ has the lowest MPC, and will thus be rejected prior to 


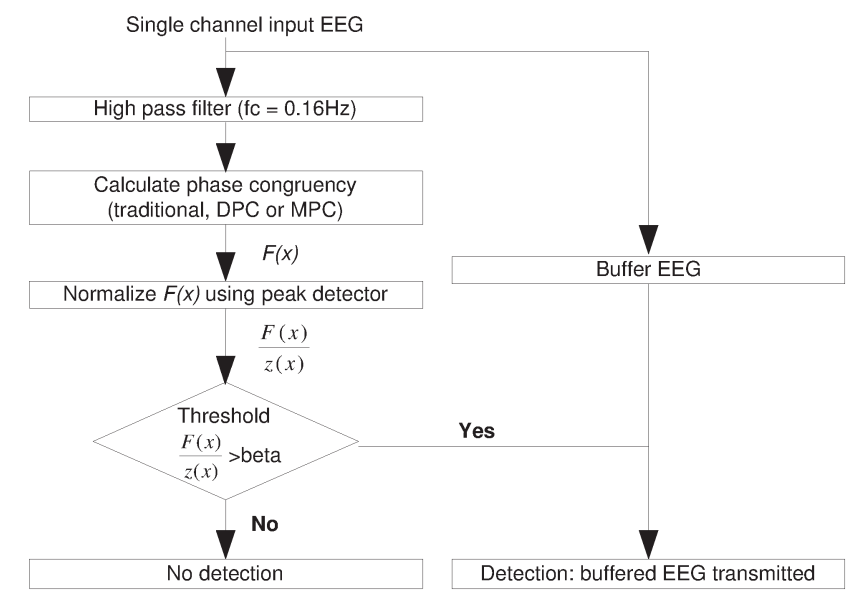

Fig. 3. Data selection algorithm to identify interictal spikes

transmission. A data selection algorithm is now designed to exploit this difference in modified phase congruency, in order to identify candidate interictal activity.

\section{E. Proposed data selection algorithm}

A flowchart of the proposed data selection algorithm is shown in Fig. 3. Initially, the input EEG is fed sample-bysample into a buffer of duration equal to half the recording window. Hence, if the current sample is detected as a candidate event, half the recording window of data before the candidate event may be transmitted and the transmitter would remain on for a further half recording window duration. The duration of the recording window should ideally be selected by a neurologist. For analysis, $1 \mathrm{~s}, 2.5 \mathrm{~s}, 5 \mathrm{~s}$ and $10 \mathrm{~s}$ windows have been simulated. Note that the recording window of data is selected after a detection has occurred, hence all calculations are on a sample-by-sample basis unless otherwise specified.

Simultaneously, the input EEG signal is filtered using a first order high pass filter with cut-off at $0.16 \mathrm{~Hz}$ (as recommended in [19]). In most EEG systems [20] [21], a first order filter is implemented to ac couple the input signal. Thus the filter implemented here replicates this hardware filter for signals obtained from dc coupled EEG systems.

Phase congruency (either traditional, DPC or MPC) is then calculated for every time sample $x$. As discussed before, spikes are expected to have higher phase congruency than background. Hence a fixed threshold is applied as the last step of the algorithm, to distinguish between them.

Prior to applying a fixed threshold, the calculated phase congruency feature $F(x)$ must be normalized to restrict its value between $\{0,1\}$. This normalization is carried out using a peak detector. For each recording, the peak detector is first set to the initial value of the feature at a time sample $x=0$ and stores this value as $z(x)$. At a later time $x+\delta$, if the calculated feature $(F(x+\delta))$ exceeds $z(x)$, then $z(x+\delta)$ is updated to have the value of the former (for example $z(x+\delta)=F(x+$ $\delta)$ ). Otherwise, $z(x+\delta)=z(x)$. The calculated feature $F(x)$ is then divided by $z(x)$ and the result is thresholded. If the normalized feature exceeds the threshold $\beta$, a detection occurs and the recording window of data is transmitted. If the normalized feature falls below $\beta$, there is no detection.

\section{F. Test database}

The data selection algorithm has been tested on 40 data sections across 10 EEG channels: F7, F8, FP1, FP2, O1, O2, T3, T4, T5 and T6. The total duration of the data was over 103 hours and contained 992 expert marked interictal spikes from 25 randomly chosen adults. The data is sampled at either $200 \mathrm{~Hz}$ or $256 \mathrm{~Hz}$. Test data has been recorded at the National Society of Epilepsy (NSE) UK, during routine, long term and ambulatory monitoring. Channels were recorded from scalp EEG electrodes setup in a referential montage and have been analyzed in the same montage. Artifacts have not been removed from the data, hence the database contains not only muscle artifacts but many others caused by eye blink and eye movement, walking, mouth and limb movement, calibration, fixing electrodes and line noise.

Interictal spikes have been marked by neurologists and EEG technicians at the NSE. Of the 27 data sections that contained spikes, there is a significant difference in the number of spikes between data sections (or tests), ranging from 1 to 644 . All tests have duration greater than 10 minutes, with mostly 2 hours recordings and two tests each lasting over 21 hours.

\section{Performance analysis}

\section{A. Metrics}

Sensitivity is defined as the number of correctly detected events or true positives $C_{i}$ for a data section $i$, as a fraction of the total number of expert marked events $N_{i}$. Hence the arithmetic mean of the sensitivity [22] across $M$ tests is

$$
\text { Mean sensitivity }=\frac{1}{M} \sum_{i=1}^{M} \frac{C_{i}}{N_{i}} \times 100 \%
$$

However, computing the arithmetic mean of the individual sensitivity values does not account for the difference in the number of spikes and duration between tests. To incorporate this difference, [22] recommends weighting the sensitivity of a test $i$ by its duration $T_{i}$ and the number of expert marked spikes $N_{i}$. This weight emphasizes the importance of detecting events in records with only a few expert marked spikes and in long records. This is because long term monitoring is generally carried out on patients with infrequent epileptiform activity and high data reduction is crucial to allow the battery to last longer. The time/event sensitivity is given by

$$
\text { Time/event sensitivity }=\frac{1}{\sum_{i=1}^{M} \frac{T_{i}}{N_{i}}} \sum_{i=1}^{M} \frac{C_{i}}{N_{i}} \frac{T_{i}}{N_{i}} \times 100 \%
$$

The sensitivity of the algorithm is traded-off with the percentage of data transmitted, where the latter is defined as the duration selected for transmission as a fraction of the total duration of the record. The duration of detected events is equal to the recording window duration multiplied by the number of detected events, assuming events are spaced out within the record. The arithmetic mean of the data transmitted for each test is then calculated. 


\section{B. Results}

The data selection algorithm in Fig. 3 is simulated for a range of thresholds $\beta$, to obtain different percentage sensitivity and percentage data transmitted values. Changing $\beta$ from zero to one gives sensitivity and data transmitted values between $100 \%$ and $0 \%$ respectively. This can be plotted as a receiver operating characteristic (ROC) curve as shown in Fig. 4. When $\beta=1$, no events will be detected hence the sensitivity and data transmitted will be zero. When $\beta$ is set to zero, every sample will be detected giving $100 \%$ sensitivity and data transmitted. The threshold is varied in steps of 0.05 to obtain the trade-off points in-between.

ROC curves for traditional (PC), denoised (DPC) and modified phase congruency (MPC) algorithms are shown in Fig. 4, Fig. 5 and Fig. 6 respectively. In each figure, time/event sensitivity and mean sensitivity have been plotted against the mean data transmitted. From these figures, the following initial conclusions can be obtained:

1) $P C$ : The mean sensitivity in Fig. 4(b) is similar to chance performance across all recording windows, whilst the time/event sensitivity in Fig. 4(a) shows a worse than chance performance for $5 \mathrm{~s}, 2.5 \mathrm{~s}$ and $1 \mathrm{~s}$ recording windows. Hence it is clear that traditional phase congruency is useless as a feature to identify interictal spikes from background EEG. Comparing the two sensitivity metrics suggests that records with fewer spikes or long duration (corresponding to a higher time/event weighting) perform worse than the other records.

The time/event sensitivity of the $10 \mathrm{~s}$ recording window is much better than the other recording windows. This is due to the detection of one spike between about $12 \%$ and $40 \%$ sensitivity from a record with a single spike and long duration (more than 2 hours), and hence the record has a high time/event weighting. It is thus inferred that such detections or lack of detection of a few events could lead to a drastic change in the time/event sensitivity, hence the mean sensitivity has also been reported.

2) $D P C$ : With reference to Fig. 5, it is clear that denoised phase congruency performs better than a random detector. At $80 \%$ time/event sensitivity, more than $45 \%$ data reduction can be achieved. Higher sensitivity of $90 \%$ can be achieved for a lower data reduction of $34 \%$. The performance measured in terms of mean sensitivity is worse, with $30 \%$ to $40 \%$ data reduction at $80 \%$ sensitivity.

3) MPC: Fig. 6 shows that $50 \%$ data reduction can be achieved for more than $80 \%$ time/event sensitivity and mean sensitivity across $1 \mathrm{~s}, 2.5 \mathrm{~s}$ and $5 \mathrm{~s}$ recording windows. For the same recording windows, $90 \%$ sensitivity would increase the data transmitted to about $60 \%$.

Both sensitivity metrics in Fig. 6(a) and Fig. 6(b) show better performance for MPC than denoised phase congruency. There is an average improvement over DPC of about $4 \%$ data reduction for time/event sensitivity above $50 \%$. For mean sensitivity above $50 \%$, there is an average improvement over DPC of about $13 \%$ data reduction.

In terms of the operating point of the algorithm at a preselected threshold, the ideal operating point would be the highest sensitivity for the lowest data transmitted which is the top left corner of the ROC curve. However, the actual operating point would depend on the required sensitivity or data transmitted and would be trade-off based where higher sensitivities will come at the cost of increased data transmitted.

\section{COMPARISON WITH ALTERNATE METHODS}

To compare the performance of the ROC curves, the area under ROC curve (AUC) is often calculated [23], [24]. AUC is a single calculated summary of the performance of an algorithm. The area under the ROC curve can also be thought as the average sensitivity for all values of data transmitted or vice versa [23]. It can be compared to an ideal data selection algorithm which would have an $\mathrm{AUC}=1$, or a random or chance algorithm with an AUC $=0.5$. Thus, an algorithm with higher AUC will have an overall better performance than an algorithm with lower AUC. Table I shows the area under the mean sensitivity ROC curves (Fig. 4(b), Fig. 5(b) and Fig. 6(b)) calculated using trapezoidal estimation.

TABLE I

AREA UNDER ROC CURVE OF PC, DPC AND MPC ALGORITHMS

\begin{tabular}{c|ccc}
\hline Recording window (s) & PC & DPC & MPC \\
\hline 1 & 0.42 & 0.65 & 0.74 \\
2.5 & 0.44 & 0.65 & 0.75 \\
5 & 0.48 & 0.62 & 0.73 \\
10 & 0.54 & 0.61 & 0.69 \\
\hline
\end{tabular}

It is evident from the ROC curves in Section III-B and the AUC values in Table I that modified phase congruency has a better overall performance than the state-of-the-art denoised phase congruency. Table I also shows that traditional phase congruency performs better than chance only for the $10 \mathrm{~s}$ window.

However, when this data selection algorithm is to be implemented in hardware, a single threshold will be selected to achieve the required percentage sensitivity or percentage data transmitted. Hence it would make sense for the algorithm with the better performance at the required sensitivity and the corresponding data transmitted (or vice versa) to be chosen. This raises a few questions: how do we compare the performance of different algorithms at the required sensitivity or data transmitted? Is it possible to incorporate the difference in performance across different tests? And finally, is there a significant difference between denoised and modified phase congruency at this selected threshold?

To compare the performance of MPC and DPC when implemented in hardware, it is necessary to select thresholds $\beta_{M}$ and $\beta_{D}$ for each algorithm respectively. To achieve a mean sensitivity of about $80 \%$ for a 5 s recording window, the corresponding sensitivities and data transmitted are: $84 \%$ sensitivity for $52 \%$ data transmission for MPC and $82 \%$ sensitivity and $65 \%$ data transmission for DPC. Both algorithms do not have a common sensitivity or data transmitted, hence the comparison is not trivial. It would be possible to sweep $\beta$ in smaller steps to obtain a common sensitivity or data transmitted. However, this poses two problems: finer $\beta$ values take longer to process and do not guarantee a common point; and only comparing this common value would not give any information on the performance variation across different tests. 


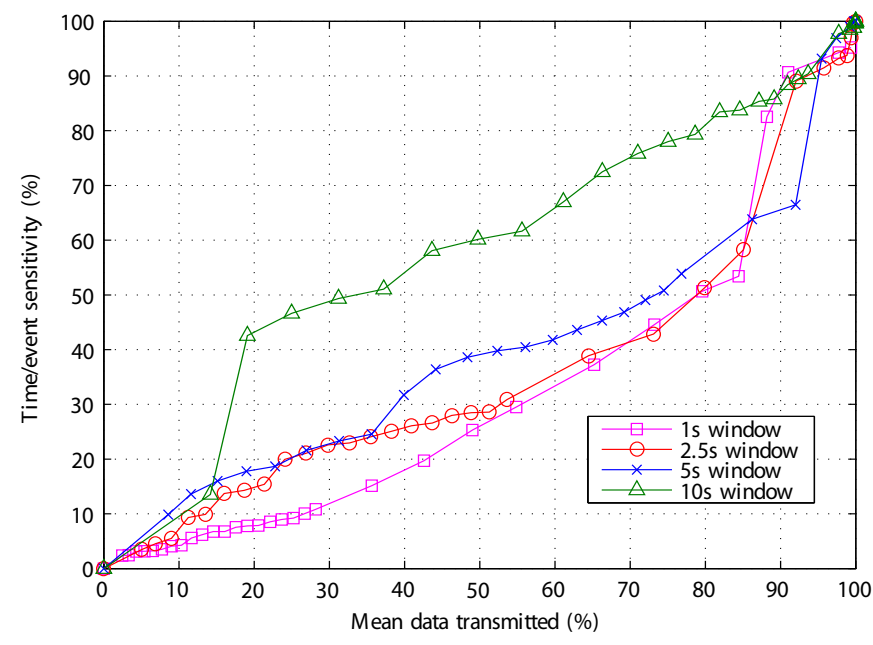

(a) Time/event sensitivity vs data transmitted

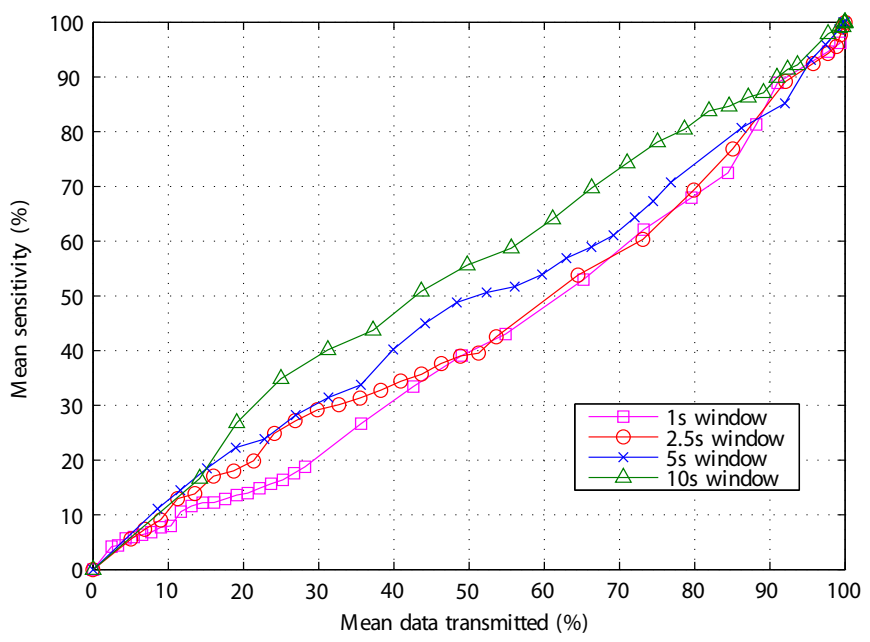

(b) Mean sensitivity vs data transmitted

Fig. 4. Performance of traditional phase congruency when computed for time/event sensitivity and mean sensitivity

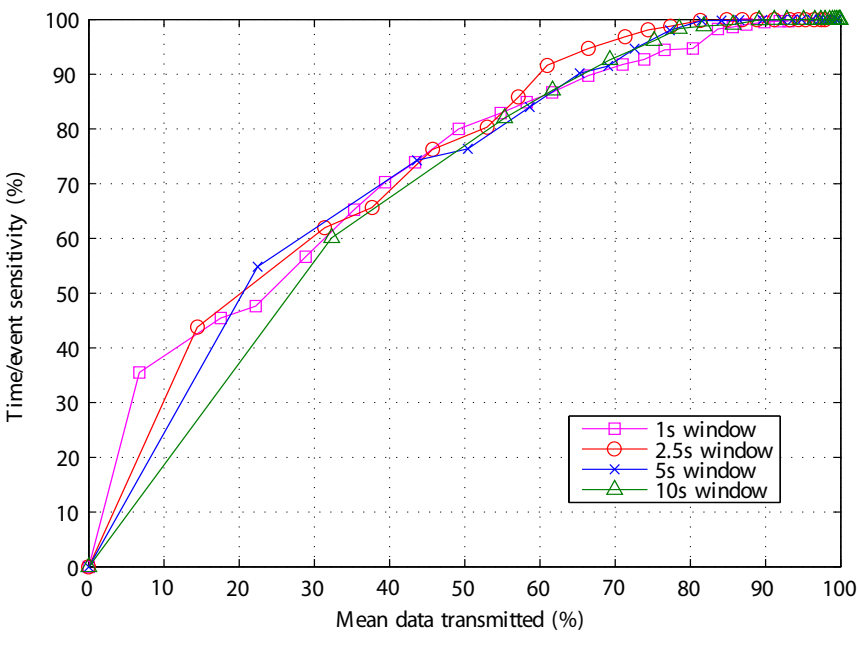

(a) Time/event sensitivity vs data transmitted

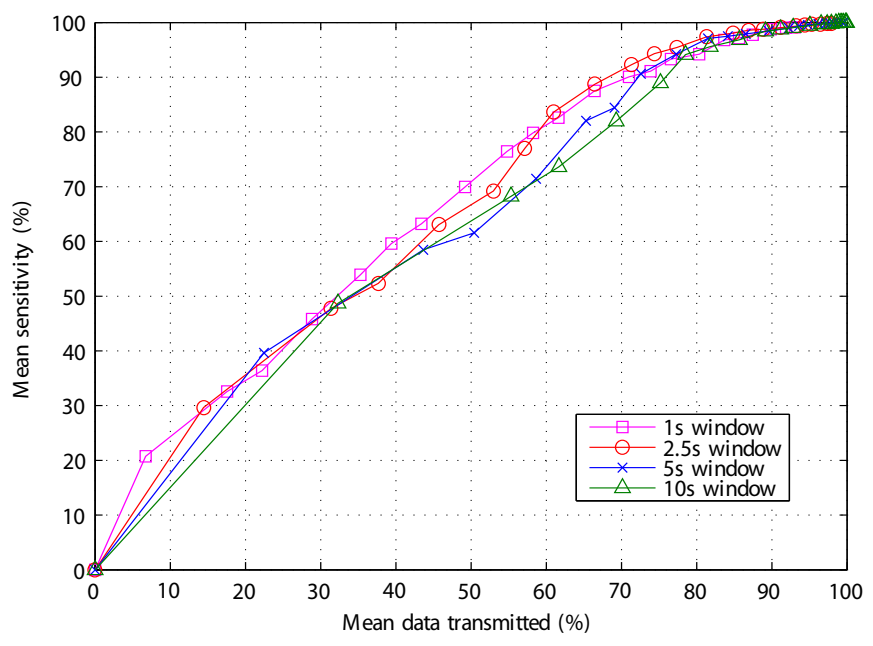

(b) Mean sensitivity vs data transmitted

Fig. 5. Performance of denoised phase congruency when computed for time/event sensitivity and mean sensitivity

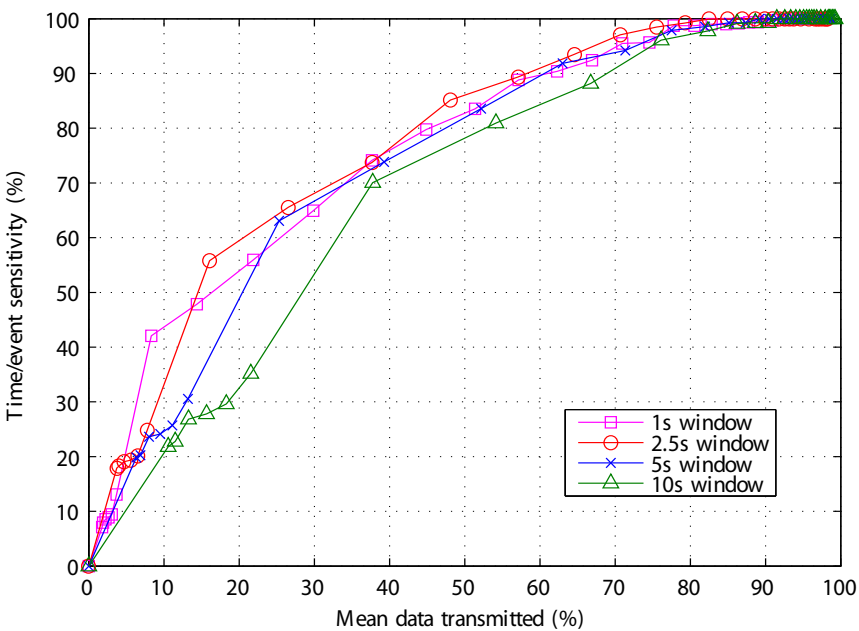

(a) Time/event sensitivity vs data transmitted

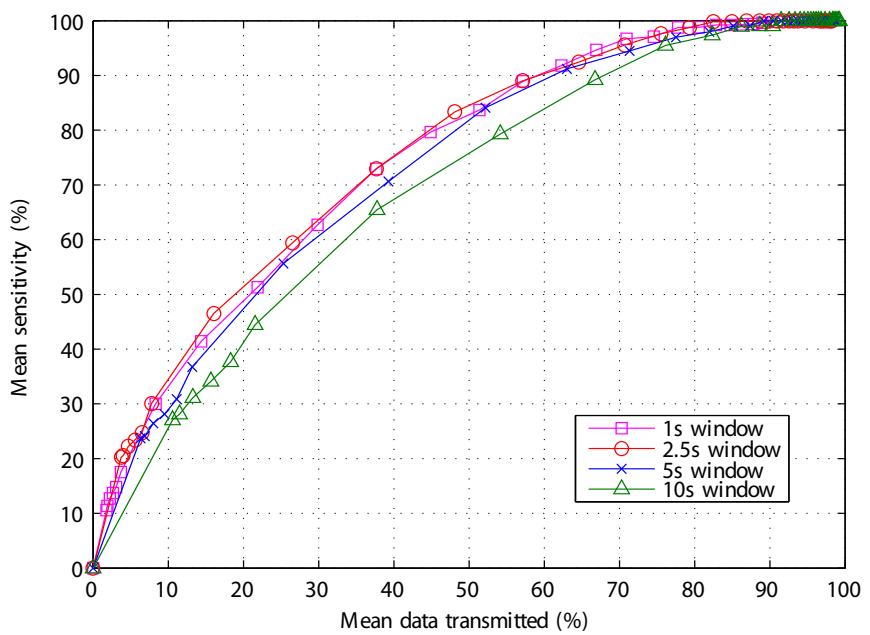

(b) Mean sensitivity vs data transmitted

Fig. 6. Performance of modified phase congruency when computed for time/event sensitivity and mean sensitivity 


\section{A. Variation between tests}

To address the variation in algorithm performance across different tests, at the same thresholds $\beta_{M}$ and $\beta_{D}$, the sensitivity and data transmitted for the individual tests containing interictal spikes, have been plotted in Fig. 7. Each test is plotted as a coordinate $(D(i), S(i))$ where $D(i)$ is the data transmitted for a test $i$ and $S(i)$ is the corresponding sensitivity. A maximum-minimum contour has also been drawn for each algorithm to show the bounds of its performance. From Fig. 7, it is clear that there is a significant performance variation between tests. The performance of the same test also differs when simulated for DPC and MPC algorithms, as the two algorithms do not have overlaying results.

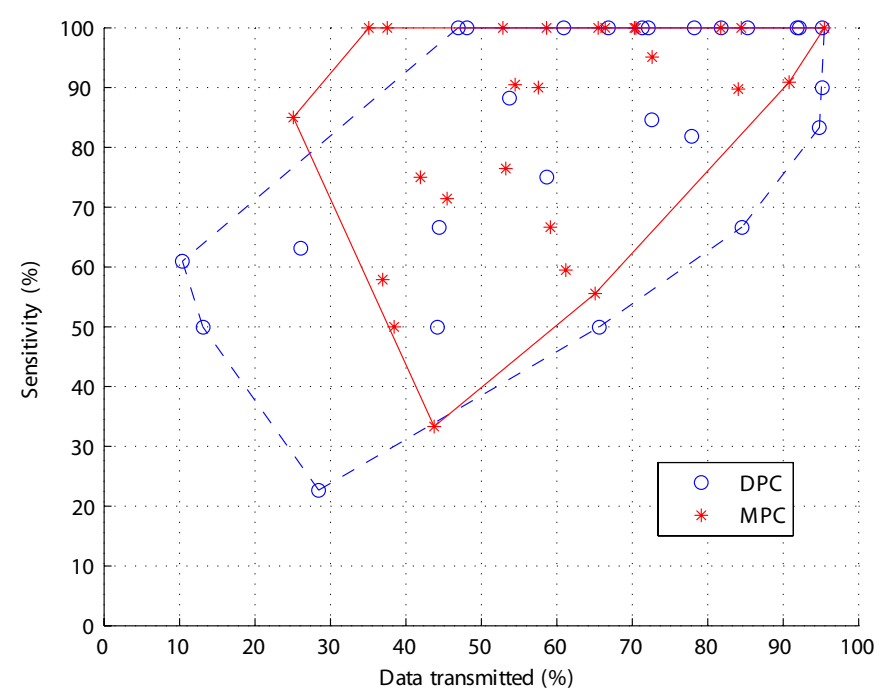

Fig. 7. Sensitivity and data transmitted for each test for denoised phase congruency ' $O$ ' and modified phase congruency '*'

\section{B. Statistical validation}

When comparing the two algorithms at a pre-selected threshold, calculating the average across all tests for sensitivity or data transmitted will not provide any information on the performance variation across tests, as discussed above. To compare both sensitivity and data transmitted for each test, lets first simplify the performance coordinate $(D(i), S(i))$ into a single value, hereon referred to as the non-ideal distance $(L)$. This is the minimum distance between $(D(i), S(i))$ and the ideal transmission coordinate $\left(I_{x}, I_{y}\right)$. The ideal transmission $\left(I_{x}, I_{y}\right)$ for a test containing spikes would be $100 \%$ sensitivity for almost $0 \%$ data transmitted assuming events are rare, giving coordinate $(0,100)$. Ideal transmission for a test without events would be $0 \%$ sensitivity for $0 \%$ data transmitted and would give $(0,0)$. Consequently, a good data selection algorithm will minimize the distance to the ideal transmission coordinate, $L$,

$$
L=\sqrt{\left(I_{y}-S(i)\right)^{2}+\left(I_{x}-D(i)\right)^{2}}
$$

From Section III-B, it is known that MPC performs better overall than DPC. Hence it can be predicted that the nonideal distance for many tests analyzed using DPC (L1) would be longer than the corresponding MPC result ( $L 2)$. To conclude whether there is a significant different between the two algorithms at the pre-selected threshold, the non-parametric Wilcoxon ranked sum statistical method for paired samples, is applied to test the following null hypothesis:

"There is no tendency for L1 to exceed L2"

The Wilcoxon test calculates the difference between matched pairs ( $L 1$ and $L 2$ ) as shown in Table II. For all 40 tests, the difference ( $L 1-L 2)$ is ranked according to their magnitude and the ranks classified as a negative or positive difference [25]. In order to compare the sum of the ranks $(W)$ to a normal distribution with mean 0 and variance 1 , we compute

$$
z=\frac{W}{S D_{W}}
$$

where $S D_{W}$ is the standard deviation of W [25]

$$
S D_{W}=\sqrt{\frac{N(N+1)(2 N+1)}{6}}
$$

and $N=40$ for 40 data sections, thus giving $S D_{W}=148.7952$. From Table II $W=482$ therefore $z=3.2394$. The calculated test statistic $z$ can be compared to the normal distribution to find a significant probability $p$ and the corresponding critical value $z_{\text {crit }}$, at which the null hypothesis may be evaluated. For a one-tailed distribution, at $p=0.005$, the corresponding critical value is $z_{\text {crit }}=2.576$ [25]. As $z$ is greater than $z_{\text {crit }}$, the null hypothesis is rejected. It is hence concluded that the non-ideal distance $L 1$ exceeds $L 2$ at a test significance of $0.5 \%$. This means that at about $80 \%$ mean sensitivity, there is less than $0.5 \%$ possibility of DPC (corresponding to $L 1$ ) outperforming MPC (L2).

TABLE II

PERFORMANCE OF DPC AND MPC ON EACH DATA SECTION

\begin{tabular}{c|ccc|c}
\hline Test & DPC(L1) (\%) & MPC(L2) (\%) & L1-L2 (\%) & Rank \\
\hline 1 & 95.1898 & 95.4560 & -0.2662 & -1 \\
2 & 26.3260 & 25.9996 & 0.3264 & 2 \\
3 & 63.8054 & 63.0663 & 0.7391 & 3 \\
4 & 85.2967 & 84.4454 & 0.8513 & 4 \\
$:$ & $:$ & $:$ & $:$ & $:$ \\
$:$ & $:$ & $:$ & $:$ & $:$ \\
40 & 96.6820 & 4.2221 & 92.4599 & 40 \\
\hline & \multicolumn{4}{r}{$\operatorname{Rank} \operatorname{sum}(\mathrm{W})$} \\
\hline
\end{tabular}

\section{Comparison with related work}

The proposed data selection algorithm based on computing modified phase congruency, is proven to outperform the stateof-the-art denoised phase congruency technique, when used to identify interictal spikes in EEG signals. Furthermore, the performance of modified phase congruency is compared to published spike detection algorithms from other research groups in Table III. The mean sensitivity and false positive rate of the proposed algorithm has been calculated for the $10 \mathrm{~s}$ recording window. All the algorithms listed in the most comprehensive and up-to-date review paper of spike detection algorithms published in 2009 [26], together with algorithms published since then, have been considered in order to generate 
TABLE III

PERFORMANCE COMPARISON WITH PUBLISHED SPIKE DETECTION ALGORITHMS

\begin{tabular}{ccc}
\hline Author & Sensitivity (\%) & FP/min \\
\hline Hostetler [27] & 76 & 5.20 \\
Senhadji [28] & 86 & 6.80 \\
Goelz [29] & 84 & 6.59 \\
Sugi [30] & 37 & 16.00 \\
Lucia [31] & 65 & 6.00 \\
\hline Proposed method & 80 & 3.20 \\
\hline
\end{tabular}

this table. Out of those, only algorithms with results reported from separate development and test databases of scalp EEG data obtained from adult patients have been included. Also publications for which not enough information had been reported to compute the sensitivity and false positives per minute have been excluded. Furthermore, as the proposed algorithm has been designed aiming for a potential low power real time implementation, published algorithms based on neural networks, template matching and computationally complex expert systems and association rule based classifiers have also been excluded. Considering these criteria, it can be seen in Table III that the proposed method outperforms comparable previously published spike detection algorithms. However, it is worth stressing that the comparison has not taken into account all algorithms previously reported in literature, but only those that would be suitable for low power implementation since these are the ones that are relevant in the context of this work.

The modified phase congruency based algorithm presented in this paper compliments our previous work in [24], which is based on calculating wavelet power to identify interictal spikes in scalp EEG signals. It is possible to combine both algorithms in order to achieve superior performance. However, in the authors' opinion, it is essential to first carry out a customized ultra low power ASIC implementation of both algorithms before adding any extra complexity to achieve additional data reduction.

\section{DISCUSSION}

Phase congruency, as presented here, appears to be a promising technique when power is a constraint, because Log Gabor filters can potentially be approximated in the frequency domain by bandpass filter functions. Additionally, the frequency range required for this application $(1.5 \mathrm{~Hz}$ to $64 \mathrm{~Hz}$ ) is very low, which translates to low passbands for these filters and hence lower biasing currents and power consumption. Recent circuit design techniques have proven that if an analog based design approach is chosen to implement bandpass filters with similar specifications, power levels can be within the nano-Watt range [32]-[34].

The paper also presents a novel statistical method that allows a trade-off based comparison of two algorithms which have been tested on the same database, at a fixed threshold. This characterization can be important when dealing with strict power budgets in an optimized hardware implementation, because generally the threshold will have to be pre-selected to achieve the required sensitivity or data transmitted. The commonly used method based on reporting the area under the ROC curve or mean sensitivity and data transmission is not adequate to quantify differences between algorithms because it represents an average value of performance. As illustrated in Fig. 7, for EEG signals there is a significant performance variation across records for different patients. Hence it would be more useful to know when selecting an algorithm for hardware implementation, how often algorithm A outperforms algorithm B at the pre-selected threshold (as given by the proposed comparison method), rather than which algorithm has a better mean performance on a specific test database. The proposed method incorporates this variation in performance across tests, by using the Wilcoxon matched pairs test to analyze the non-ideal distances (derived from the sensitivity and data transmitted) of each record across both algorithms. Thus, for two algorithms A and B, the Wilcoxon rank sum is weighted according to the number of times A outperforms B (or vice versa), and the absolute difference of their non-ideal distances at each test. This implies that for algorithm A to be significantly better than algorithm B, A should perform better than $\mathrm{B}$ at most tests, and with a large margin. An additional advantage of directly comparing both algorithms for each record is that significant line noise or incorrect event markers would also not represent a problem, since both algorithms will perform badly in these records and will not affect the rank sum. However, it is also worth noticing that, should configurable thresholds be an option in implementation, the proposed method should be used in addition to comparing the overall performance using, for example, (partial) area under the ROC curve.

\section{Conclusions}

A novel data selection algorithm based on phase congruency has been proposed to identify interictal spikes in scalp EEG data. The traditional method to calculate phase congruency has been implemented, but performs worse than a chance detector. The state-of-the-art method to denoise phase congruency gives $80 \%$ mean sensitivity for about 30\%-40\% data reduction, depending on the duration of the recording window. The proposed method to denoise phase congruency by dynamically estimating and compensating for muscle activity in the EEG, further improves the performance of the data selection algorithm to $80 \%$ sensitivity for more than $50 \%$ data reduction.

Both algorithms have been compared using the area under the ROC curve, together with a novel statistical validation method based on using the Wilcoxon matched pairs test. The algorithm comparison methodology proposed in this work, incorporates performance variation across different data sections at a fixed threshold and helps to better understand the expected behavior of the data selection algorithm when implemented in hardware.

\section{REFERENCES}

[1] S. Sisodiya, "Etiology and management of refractory epilepsies," Nat. Clin. Pract. Neuro., vol. 3, no. 6, pp. 320-330, 2007.

[2] E. Waterhouse, "New horizons in ambulatory electroencephalography," IEEE Eng. Med. Biol. Mag., vol. 22, no. 3, pp. 74-80, 2003.

[3] R. P. Lesser, "Epilepsy: Does continuous EEG monitoring improve seizure control?" Nat. Rev. Neurol., vol. 5, no. 11, pp. 581-582, Nov. 2009.

[4] A. J. Casson, S. J. M. Smith, J. S. Duncan, and E. Rodriguez-Villegas, "Wearable EEG: what is it, why is it needed and what does it entail?" in IEEE EMBC, Aug. 2008, pp. 5867-5870. 
[5] A. J. Casson and E. Rodriguez-Villegas, "Data reduction techniques to facilitate wireless and long term AEEG epilepsy monitoring," in IEEE EMBS NER, Hawaii, May 2007, pp. 298-301.

[6] R. F. Yazicioglu, P. Merken, R. Puers, and C. Van Hoof, "A $60 \mu \mathrm{W}$ $60 \mathrm{nV} / \sqrt{\mathrm{Hz}}$ readout front-end for portable biopotential acquisition systems," IEEE J. Solid-State Circuits, vol. 42, no. 5, pp. 1100-1110, May 2007.

[7] P. Kovesi, "Image features from phase congruency," Videre: J. Computer Vision Research, vol. 1, no. 3, Summer 1999.

[8] Y. Punsawad and Y. Wongsawat, "Palmprint image enhancement using phase congruency," in IEEE ROBIO, Bangkok, Feb. 2009, pp. $1643-$ 1646.

[9] H. Zhi-Kai, L. De-Hui, Z. Wei-Zhong, and H. Ling-Ying, "Multi-view face database recognition using phase congruency and SVM classifier," in ICCEE, Phuket, Dec. 2008, pp. 219-222.

[10] Y. Wongsawat, "Epileptic seizure detection in EEG recordings using phase congruency," in IEEE EMBC, Vancouver, Aug. 2008, pp. 927930.

[11] S. Venkatesh and R. A. Owens, "An energy feature detection scheme," in Int. Conf. Image Processing, Singapore, 1989, pp. 553-557.

[12] L. Logesparan and E. Rodriguez-Villegas, "Improving phase congruency for EEG data reduction," in IEEE EMBC, Buenos Aires, Aug. 2010.

[13] A. J. Casson, D. C. Yates, S. J. M. Smith, J. S. Duncan, and E. Rodriguez-Villegas, "Wearable electroencephalography," IEEE Eng. Med. Biol. Mag., vol. 29, no. 3, pp. 44-56, 2010.

[14] J. Gotman, "Automatic detection of seizures and spikes," J. Clin. Neurophysiol., vol. 16, no. 2, pp. 130-140, Mar. 1999.

[15] I. I. Goncharova, D. J. McFarland, T. M. Vaughan, and J. R. Wolpaw, "EMG contamination of EEG: spectral and topographical characteristics," Clin. Neurophysiol., vol. 114, no. 9, pp. 1580-1593, Sep. 2003.

[16] R. D. O'Donnell, J. Berkhout, and W. R. Adey, "Contamination of scalp EEG spectrum during contraction of cranio-facial muscles," Electroencephalogr. and Clin. Neurophysiol., vol. 37, no. 2, pp. 145-151, Aug. 1974.

[17] J. Gotman, J. R. Ives, and P. Gloor, "Frequency content of EEG and EMG at seizure onset:possibility of removal of EMG artefact by digital filtering," Electroencephalogr. and Clin. Neurophysiol., vol. 52, pp. 626639, Dec. 1981

[18] P. D. Kovesi, "MATLAB and Octave functions for computer vision and image processing," School of Computer Science \& Software Engineering, The University of Western Australia, available from: $<$ http://www.csse.uwa.edu.au/ pk/Research/MatlabFns/index.html $>$.

[19] M. R. Nuwer, G. Comi, R. Emerson, A. Fuglsang-Frederiksen, J.-M. Gurit, H. Hinrichs, A. Ikeda, F. Jose C. Luccas, and P. Rappelsburger, "IFCN standards for digital recording of clinical EEG," Electroencephalogr. and Clin. Neurophysiol., vol. 106, no. 3, pp. 259-261, 1998.

[20] N. Verma, A. Shoeb, J. Bohorquez, J. Dawson, J. Guttag, and A. Chandrakasan, "A micro-power EEG acquisition SoC with integrated feature extraction processor for a chronic seizure detection system," J SolidState Circuits, vol. 45, no. 4, pp. 804-816, 2010.

[21] C. D. Binnie, A. J. Rowan, and T. Gutter, A manual of electroencephalographic technology. Cambridge: Cambridge University Press, 1982.

[22] A. J. Casson, E. Luna, and E. Rodriguez-Villegas, "Performance metrics for the accurate characterisation of interictal spike detection algorithms," J. Neuroscience Methods, vol. 177, no. 2, pp. 479-487, Mar. 2009.

[23] T. A. Lasko, J. G. Bhagwat, K. H. Zou, and L. Ohno-Machado, "The use of receiver operating characteristic curves in biomedical informatics," $J$. Biomed. Informatics, vol. 38, no. 5, pp. 404 - 415, Oct. 2005.

[24] A. J. Casson and E. Rodriguez-Villegas, "Towards online data reduction for portable electroencephalography systems in epilepsy," IEEE Trans. Biomed. Eng., vol. 56, no. 12, pp. 2816-2825, Dec. 2009.

[25] A. Delorme, "Statistical methods," Encyclopedia of Medical Device and Instrumentation, vol. 6, pp. 240-264, 2006.

[26] J. J. Halford, "Computerized epileptiform transient detection in the scalp electroencephalogram: Obstacles to progress and the example of computerized ecg interpretation," Clinical Neurophysiology, vol. 120, no. 11 , pp. $1909-1915,2009$
[27] W. E. Hostetler, H. J. Doller, and R. W. Homan, "Assessment of a computer program to detect epileptiform spikes," Electroencephalogr and Clinical Neurophysiol, vol. 83, no. 1, pp. 1-11, 1992.

[28] L. Senhadji, J. L. Dillenseger, F. Wendling, C. Rocha, and A. Kinie, "Wavelet analysis of EEG for three-dimensional mapping of epileptic events," Annals of Biomedical Engineering, vol. 23, no. 5, pp. 543-552, 1995.

[29] H. Goelz, R. Jones, and P. B. PJ, "Wavelet analysis of transient biomedical signals and its application to detection of epileptiform activity in the EEG," Clin Electroencephalogr, vol. 31, no. 4, pp. 181-191, 2000.

[30] T. Sugi, M. Nakamura, A. Ikeda, and H. Shibasaki, "Adaptive EEG spike detection: determination of threshold values based on conditional probability," Frontiers of Medical \& Biological Engineering, vol. 11, no. 4, pp. 261-277, 2001

[31] M. D. Lucia, J. Fritschy, P. Dayan, and D. Holder, "A novel method for automated classification of epileptiform activity in the human electroencephalogram-based on independent component analysis," Medical and Biological Engineering and Computing, vol. 46, no. 3, pp. 263-272, 2008

[32] S. Haddad, R. Houben, and W. Serdijn, "Analog wavelet transform employing dynamic translinear circuits for cardiac signal characterization," in ISCAS, May 2003, pp. 121-124.

[33] P. Agostinho, S. Haddad, J. De Lima, and W. Serdijn, "An ultra low power CMOS $\mathrm{pA} / \mathrm{V}$ transconductor and its application to wavelet filters," Analog Integrated Circuits and Signal Processing, vol. 57, no. 1, pp. 19-27, 2008.

[34] L. Hongmin, H. Yigang, and Y. Sun, "Detection of cardiac signal characteristic point using log-domain wavelet transform circuits," Circuits, Systems, and Signal Processing, vol. 27, no. 5, pp. 683-698, 2008.

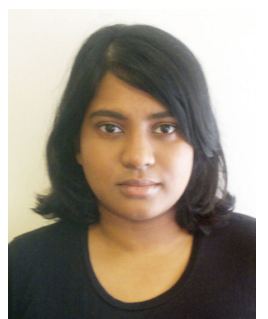

Lojini Logesparan (S'10) received the B.Eng. degree in Electrical and Electronic Engineering from Imperial College London, and the Associateship of the City and Guilds Institute, in 2007. She is currently working towards a Ph.D. in low power circuits and systems design at Imperial College London, where her work focuses on algorithm and circuit design for online EEG data reduction.

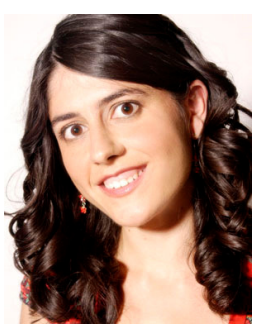

Esther Rodriguez-Villegas (SM'08) received the M.Sci. and Ph.D. degrees from the Department of Physics, University of Seville, Seville, Spain, in 1996 and 2002, respectively. From 1999 to September 2002 she was an Assistant Professor with the University of Seville, and in 2000 she started a collaboration with the Circuits and Systems Group, Imperial College London, London, U.K., being involved in biomedical projects. She is currently a Senior Lecturer (Associate Professor) with the Department of Electrical and Electronic Engineering, Imperial College London, where she specializes in ultra-low-power electronic circuits and systems for truly wearable physiological monitoring. She is the author of more than 100 peer-reviewed papers and a book on FGMOS transistors which was published by the Institution of Engineering and Technology (IET) in 2006.

Dr. Rodriguez-Villegas has been a member of technical committees in several international conferences such as the IEEE International Symposium on Circuits and Systems, the IEEE International Conference on Electronics, Circuits and Systems, and the IEEE Biomedical Circuits and Systems (BioCAS). In 2010 she organized a mini-symposium on truly wearable medical devices at the IEEE Engineering in Medicine and Biology Conference. In 2009 she was awarded the Complutense Young Award for Science and Technology, and an IET innovation award. 